\title{
BiomarCaRE: rationale and design of the European BiomarCaRE project including 300,000 participants from 13 European countries
}

\author{
Tanja Zeller • Maria Hughes - Tarja Tuovinen • Arne Schillert • Annette Conrads-Frank • \\ Hester den Ruijter • Renate B. Schnabel · Frank Kee • Veikko Salomaa • \\ Uwe Siebert · Barbara Thorand · Andreas Ziegler · Heico Breek • \\ Gerard Pasterkamp $\cdot$ Kari Kuulasmaa $\cdot$ Wolfgang Koenig $\cdot$ Stefan Blankenberg
}

Received: 24 February 2014 / Accepted: 2 September 2014/Published online: 20 September 2014

(C) The Author(s) 2014. This article is published with open access at Springerlink.com

\begin{abstract}
Biomarkers are considered as tools to enhance cardiovascular risk estimation. However, the value of biomarkers on risk estimation beyond European risk scores, their comparative impact among different European regions and their role towards personalised medicine remains uncertain. Biomarker for Cardiovascular Risk Assessment in Europe (BiomarCaRE) is an European collaborative research project with the primary objective to assess the value of established and emerging biomarkers for cardiovascular risk prediction. BiomarCaRE integrates
\end{abstract}

On behalf of the BiomarCaRE consortium.

Tanja Zeller and Maria Hughes have contributed equally to this work.

Electronic supplementary material The online version of this article (doi:10.1007/s10654-014-9952-x) contains supplementary material, which is available to authorized users.

T. Zeller $(\bowtie) \cdot$ R. B. Schnabel · S. Blankenberg $(\bowtie)$ Clinic for General and Interventional Cardiology, University Heart Centre Hamburg, Martinistr. 52, 20246 Hamburg, Germany

e-mail: t.zeller@uke.de

S. Blankenberg

e-mail: s.blankenberg@uke.de

T. Zeller · M. Hughes - A. Schillert · R. B. Schnabel ·

A. Ziegler $\cdot$ S. Blankenberg

German Centre for Cardiovascular Research (DZHK), Partner

Site Hamburg/Lübeck/Kiel, Hamburg, Germany

\section{Hughes - F. Kee}

UK Clinical Research Collaboration Centre of Excellence for Public Health, Queens University of Belfast, Belfast,

Northern Ireland, UK

T. Tuovinen · V. Salomaa $\cdot$ K. Kuulasmaa

National Institute for Health and Welfare, Helsinki, Finland clinical and epidemiological biomarker research and commercial enterprises throughout Europe to combine innovation in biomarker discovery for cardiovascular disease prediction with consecutive validation of biomarker effectiveness in large, well-defined primary and secondary prevention cohorts including over 300,000 participants from 13 European countries. Results from this study will contribute to improved cardiovascular risk prediction across different European populations. The present publication describes the rationale and design of the BiomarCaRE project.

Keywords BiomarCaRE - Biomarker - Cardiovascular Risk Assessment $\cdot$ MORGAM $\cdot$ EU

A. Schillert · A. Ziegler

Institut für Medizinische Biometrie und Statistik, Universität zu Lübeck, Universitätsklinikum Schleswig-Holstein, Campus

Lübeck, Lübeck, Germany

A. Conrads-Frank - U. Siebert

Department of Public Health and Health Technology

Assessment, UMIT - University for Health Sciences, Medical

Informatics and Technology, Hall i.T., Austria

H. d. Ruijter · G. Pasterkamp

Universitair Medisch Centrum, Utrecht, The Netherlands

U. Siebert

Harvard School of Public Health, Harvard Medical School,

Boston, MA, USA

B. Thorand

Institute of Epidemiology II, Helmholtz Zentrum München, German Research Center for Environmental Health, Neuherberg, Germany 


\section{Introduction}

Despite significant advances in treatment, cardiovascular disease (CVD) remains the leading cause of death worldwide. Traditional risk factors including dyslipidemia, hypertension, diabetes, obesity, and smoking account for a large proportion of the risk of myocardial infarction and stroke globally [36]. Most of these traditional risk factors are modifiable and their management is likely to reduce risk of CVD in both primary and secondary prevention settings [12]. However, risk estimates based on these classical risk factors only partially explain CVD incidence in the general population and risk estimates vary across European populations. Despite the availability of various global risk assessment scores like the Framingham Score [7], the PROCAM Score [3] and the European Society of Cardiology SCORE [6], prediction of cardiovascular events is incomplete and a considerable number of patients at risk go unidentified on the basis of traditional risk factors alone. Khot et al. [18] have reported that $62 \%$ of patients with MI present with none or only one risk factor and less than $10 \%$ show three or four risk factors. To improve risk estimation above and beyond traditional risk scores, and to improve therapy decision making and guidance, novel (emerging) biomarkers are of considerable interest [4]. A number of those biomarkers are thought to provide additional prognostic information; however, only few studies have evaluated a large panel of biomarkers [4]. Therefore, it is not clear whether these proposed biomarkers might merely be proxies for known risk factors and therefore whether they really provide incremental predictive and discriminatory information. Most importantly, are there so far undetected novel biomarkers that might perform even better than known biomarkers?

Molecular omics-based approaches such as profiling of microRNAs, the proteome of microparticles, the transcriptome and the metabolome represent new and promising approaches for the discovery of novel biomarkers and

\author{
B. Thorand \\ German Centers for Cardiovascular Research (DZHK), \\ Partner Site Munich, Munich, Germany \\ A. Ziegler \\ Zentrum für Klinische Studien Lübeck, Universität zu Lübeck, \\ Lübeck, Germany \\ H. Breek \\ Cavadis B.V., Utrecht, The Netherlands \\ W. Koenig \\ Department of Internal Medicine II-Cardiology, University of \\ Ulm Medical Centre, Ulm, Germany
}

might lead to the identification of markers which better distinguish individuals who may experience incident cardiovascular events. Overall, risk estimation needs to be assessed across populations in different European regions, as well as in the secondary prevention setting where even less is known about the performance of established and novel biomarkers for risk estimation. Ultimately, we need to know whether a modified risk estimation model will translate into a change in treatment [4].

Within the BiomarCaRE project, promising novel biomarkers will be selected in order to develop risk estimation models for incident CVD and they will be validated across European populations. Success in building a robust prediction score could greatly facilitate individualized risk estimation, screening, and diagnosis of CVD. The novel risk score would have the potential to change existing clinical guidelines and could thus have significant public health impact.

\section{The consortium}

The collaborative BiomarCaRE consortium, an FP7 funded project, integrates the efforts of 25 academic institutions and five small/medium-sized, research intensive enterprises (SMEs) with a focus on cardiovascular biomarker research across Europe (Fig. 1). BiomarCaRE brings together largescale epidemiological and clinical data and biomaterial resources across Europe and diverse expertise in epidemiology, clinical research, data and sample management, clinical chemistry, molecular biology, and biostatistics. The consortium capitalizes on in depth knowledge of omics-based candidate markers and integrates modern technologies of multiple biomarker assessment. It comprises 21 well-established prospective European population-based cohort studies, most of which were previously harmonized in the MORGAM Project [9], four cohorts of diseased subjects (disease cohorts, secondary prevention) and four clinical trials, totalling over 300,000 participants with a follow-up of over three million person years and storage of selected biomaterial of all participants in one central BiomaCaRE laboratory (University Heart Center Hamburg). This large individual-based database provides a unique opportunity to investigate the performance of established and novel biomarkers for Cardiovascular Risk Assessment across Europe.

An academic partner coordinates the consortium; the focus, however, is constructed around novel, emerging biomarkers and innovative technology and assays derived by SMEs. The consortium comprises an established research governance infrastructure, data harmonization and sharing arrangements, two central secure, access-managed phenotypic and biomarker data repositories as well as a core laboratory for biomarker measurements. 


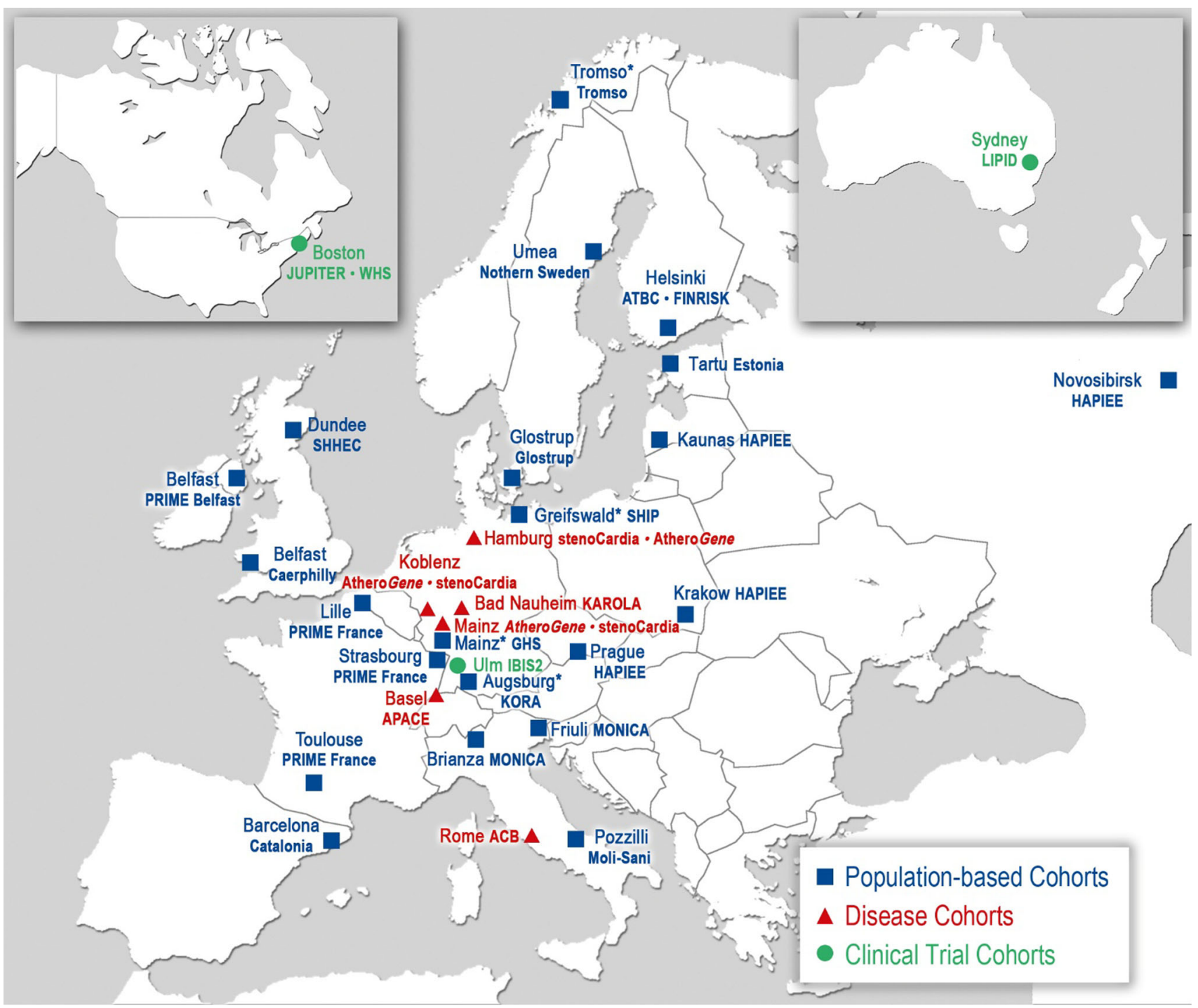

Fig. 1 BiomarCaRE collaborating countries and cohorts across Europe. Asterisk indicates cohort with imaging data available

Objective of BiomarCaRE

The objective of the BiomarCaRE consortium is to combine innovation in biomarker discovery with validation of newly identified and established biomarkers for CVD prediction across Europe. Ultimately, the BiomarCaRE consortium aims to develop a "European biomarker panel" for CVD prediction including classical risk factors and established and novel biomarkers. This will be achieved on the basis of SME-driven development of innovative technology and immunological/clinical chemistry assays and a large collaboration of well-defined European cohorts in primary and secondary prevention. The BiomarCaRE consortium measures success not only in terms of development of novel risk estimation models and their subsequent clinical utility, but also in terms of the development of novel, innovative technologies.

\section{Methods}

\section{Design of BiomarCaRE}

The BiomarCaRE project is designed as a multi-modular study including: (1) biomarker selection based on omics discovery studies as well as literature, and (2) assay development (Module 1), (3) data harmonization of largescale studies and (4) biomarker determination, analyses and validation (Module 2), and (5) biomarker assessment in clinical trials and (6) economic evaluation (Module 3). The main disease endpoints assessed in BiomarCaRE are incident acute coronary events, stroke, heart failure, atrial fibrillation, and diabetes mellitus. Other cardiovascular endpoints such as venous thrombosis will also be studied. Figure 2 gives a detailed overview about the multi-modular BiomarCaRE design. 


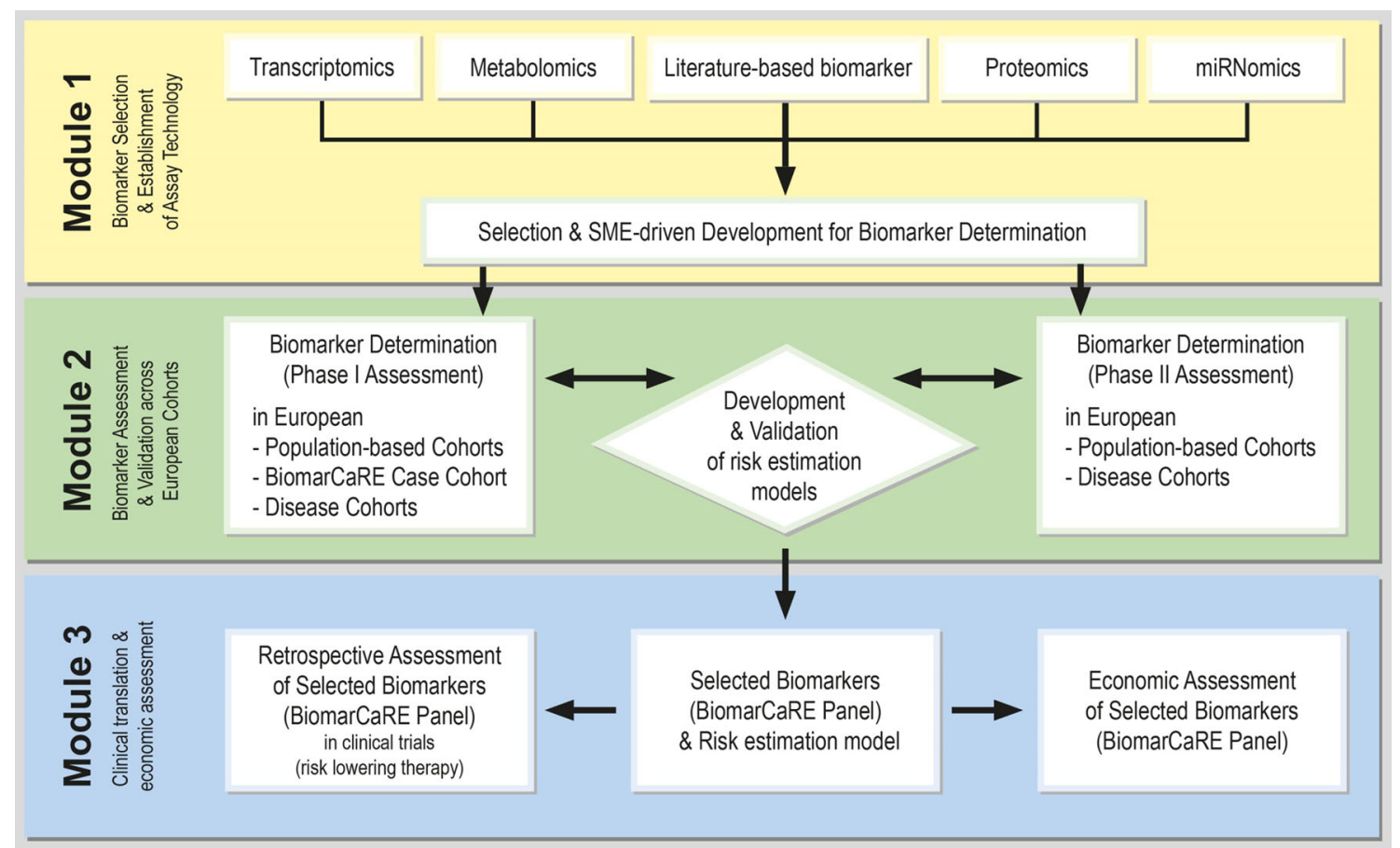

Fig. 2 BiomarCaRE multi-modular concept. From biomarker selection and assay development (Module 1) to biomarker measurements and statistical analyses (Module 2) to clinical translation and economic assessment (Module 3). Module 1: Established and emerging biomarkers are prioritised according to their association with CVD risk, novel biomarkers are selected based on pre-existing non-publically available-omics datasets. Assay development is guided by SMEs and optimised for medium to high-throughput measurement.

\section{Cohorts, variables and biomaterial in BiomarCaRE}

An overview of all cohorts, follow-up times and available biomaterial samples is outlined in Tables 1, 2, 3, 4, 5, 6 and a detailed description of the cohorts is provided in the Supplementary Material. For each cohort, ethical approval has been granted by the respective Ethical Committees and each participant gave informed consent.

\section{Population-based cohorts}

The population cohorts originate from different countries of all European regions (Fig. 1). Each cohort is based on a well-defined population. Consistent measurement and data collection procedures were used for demographic, lifestyle, biological and clinical data. Some of the cohorts have ultrasound-based imaging and magnetic resonance imaging (MRI) data available (Table 1). Overall, the general population cohorts comprise 171,000 men and 129,000 women; 45,000 of them have serially sampled biomarkers available. The mean age at baseline is 51.2 (SD 12.7) years. All cohorts include subjects in mid-life, a time when early onset CVD manifests and where it is of importance to
Module 2: The predictive value of biomarkers is assessed separately in population and disease based cohorts in a two phase approach; phase I assessment and phase II validation. Module 3 assesses the clinical utilisation of BiomarCaRE risk panels in clinical trials for their interaction with risk lowering therapy and develops a decisionanalytical model to estimate long term cost-effectiveness of a primary or secondary preventive strategy guided by biomarker testing

target improved risk estimation (Table 2). All cohorts followed-up the study participants prospectively for a range of 2.5-25 years for fatal and non-fatal acute coronary events and stroke and death. Some of the cohorts also followed-up heart failure, atrial fibrillation, type 2 diabetes, peripheral vascular disease, venous thromboembolism, and cancer.

The harmonised baseline variables include measured systolic and diastolic blood pressure, blood lipids, weight, height, waist and hip circumference, and questions on smoking, disease history and medication for hypertension, dyslipidaemia and diabetes. Assessment and validation of the end-points varied between cohorts (Kulathinal et al. [20] ). Acute coronary events are harmonized to categories: definite or possible myocardial infarction or coronary death, unstable angina pectoris, cardiac revascularization, or unclassifiable death, where the data are insufficient for the other coronary diagnoses and there is no evidence of other causes. The categories can be combined to define different end-points for analysis. Stroke has been characterized as ischaemic stroke, intracerebral haemorrhage, and subarachnoid haemorrhage, although reliable diagnostic information for the subtyping is not always available, in 


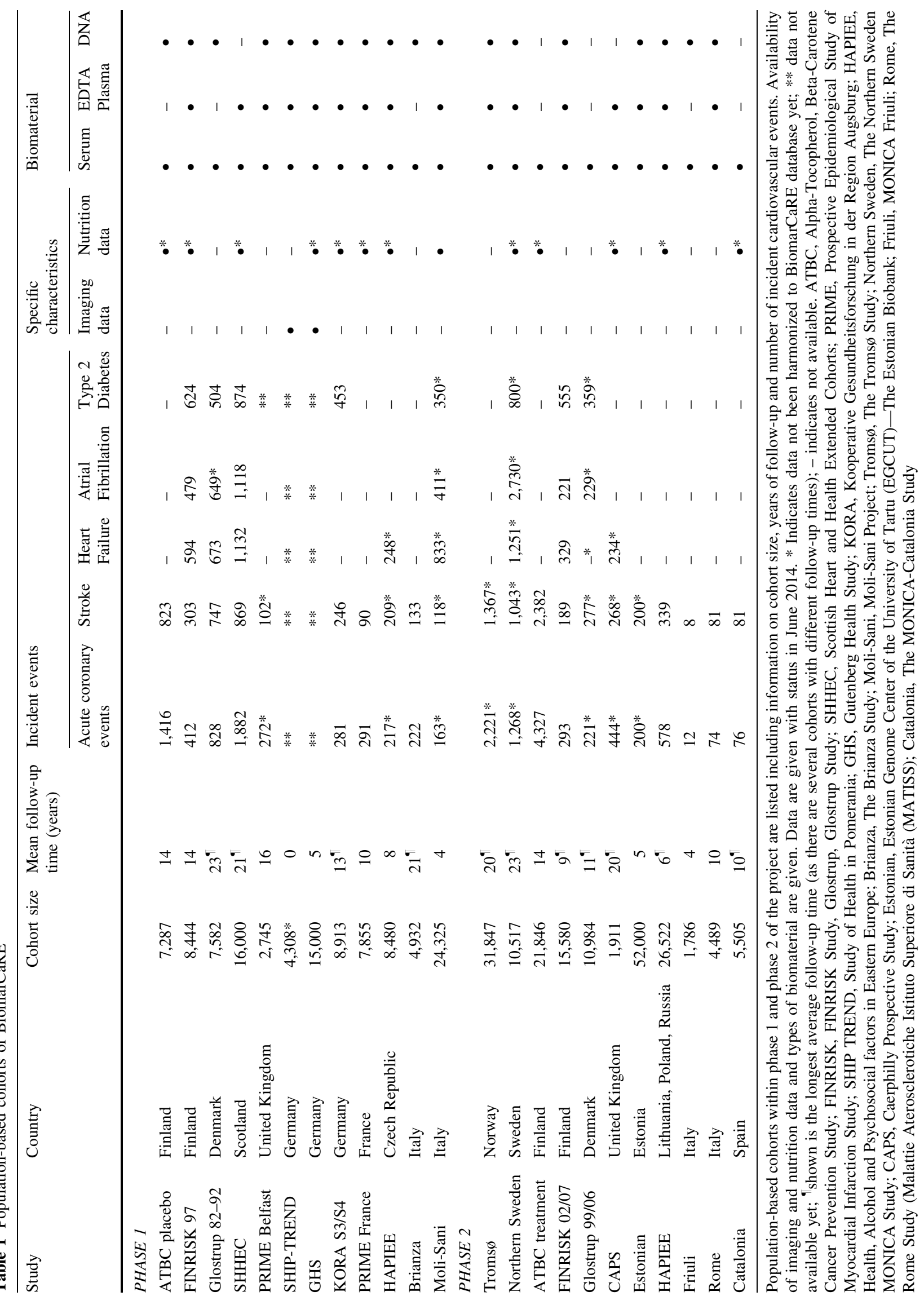


particular for the early years of follow-up. Follow-up for heart failure, atrial fibrillation, type 2 diabetes, peripheral vascular disease, venous thromboembolism and cancer were usually based on linkage with national hospitalization registers, other administrative registers and cancer registers.

\section{Disease cohorts}

In patients with manifest CVD, in particular after an acute coronary syndrome (ACS), a number of clinical variables have been proven useful for risk assessment. These variables have been integrated into various scores like the Thrombolysis in Myocardial Infarction (TIMI) Risk Score, and the Global Registry of Acute Coronary Event (GRACE) Score. Based on such parameters fairly reliable prediction can be made not only short-term but even over a period of more than 5 years [11]. However, despite such routinely available variables, further improvement of risk assessment in this high-risk group is of paramount importance because available treatment options should be tailored to the individual patient's risk. The BiomarCaRE consortium offers the opportunity to directly translate population-derived biomarker information into the clinical setting of manifest CVD and ACS. Within the consortium four disease cohorts comprising almost 9,000 individuals with either angiographically proven stable coronary artery disease (CAD) mostly after myocardial infarction or individuals presenting with suspected acute ACS have been harmonized for CVD history, clinical symptoms and classical cardiovascular risk factor information. The clinical endpoints followed up in the disease cohorts are (1) total mortality, (2) cardiac death, (3) cardiovascular death including fatal cardiovascular events such as cerebrovascular diseases, and (4) cardiovascular events including the combined endpoint of cardiovascular death and non-fatal myocardial infarction (Table 3). The mean age is 61.5 (SD 12.4) years. Follow-up varies between 6 months and 7.5-10 years. Baseline variables are outlined in Table 4. Combining data from different diseases cohorts in BiomarCaRE, in particular from the AtheroGene and KAROLA studies with long-term follow-up, guarantees sufficient power to reliably describe biomarker associations with recurrent cardiovascular events and to test the applicability of a marker panel in diverse clinical settings.

\section{Clinical trials}

Various biomarkers may be affected by contemporary pharmacological strategies as seen for example in the case of hsCRP and statin treatment [24]. This biomarker may be lowered by $40 \%$ when highly potent statins are given. Thus, it may be important to test the effects of various therapies on our finally selected biomarkers. Based on access to biobanks of several large clinical trials, BiomarCaRE will test biomarkers as measures of therapeutic response related to aspirin in women (Womeńs Health Study), LDL-Cholesterol lowering by statins (The LongTerm Intervention with Pravastatin in Ischaemic Disease (LIPID) Study and The Justification for the Use of Statins in Primary Prevention: An Intervention Trial Evaluating Rosuvastatin (JUPITER) trial), and anti-inflammatory treatment with an Lp-PLA2 inhibitor (darapladib) [Integrated Biomarker and Imaging Study-2 (IBIS-2)] (Table 5).

\section{Biomaterial}

A prerequisite for all BiomarCaRE cohorts is the availability of biomaterial samples, such as plasma, serum and genomic DNA. Furthermore, innovative biomaterial such as plaque tissue is also available in some of the BiomarCaRE cohorts. The Cohort Centres select samples from their repositories and transfer them to the BiomarCaRE central laboratory in Hamburg, Germany, where the measurement of all established and novel biomarkers is performed.

\section{Selected established, emerging, and "omics-based" biomarkers, and assay development by SMEs}

In Module 1 of the project (Fig. 2), established biomarkers are selected according to their association with the risk of CVD, and based on the expertise and knowledge of the BiomarCaRE consortium and the public domain. The BiomarCaRE consortium has already determined an extensive panel of biomarkers within the MORGAM Biomarker substudy [4]. Key established biomarkers to be addressed are high-sensitivity troponins (hsTn), natriuretic peptides, high-sensitivity C-reactive protein (CRP), lipids, and further markers of cardiomyocyte micronecrosis, inflammation, and renal function (Fig. 3). The concept of biomarkers reflecting different underlying pathways to disease progression combined in multiple biomarker risk scores has already been applied in several population-based studies [4, $14,21,25,39$ ] as well as in disease cohorts [26]. Several of those markers, in particular hsTn, hsCRP as well as growth differentiation factor 15 (GDF-15) have been proposed to guide therapy $[1,17,24]$. Commercially available assays are used to determine the levels of established biomarkers in the biomaterial of study participants.

Selection of novel biomarkers is based on pre-existing but not publicly available -omics datasets on quantitative proteomics, transcriptomics, metabolomics, and miRNomics. The emerging value of circulating microRNAs (miRNAs) and circulating cells such as microparticles as 
Table 2 Overall characteristics of the BiomarCaRE population cohorts

\begin{tabular}{|c|c|}
\hline \multicolumn{2}{|l|}{ Characteristics } \\
\hline Number of cohort studies ${ }^{\mathrm{a}}$ & 21 \\
\hline Number of individuals & 300,000 \\
\hline Years of baseline examinations & $1982-201$ \\
\hline Men $(\%)$ & $57 \%$ \\
\hline Mean age (SD) at baseline (years) & $51.2(12.7$ \\
\hline Smoker $(\%)$ & $38 \%$ \\
\hline Diabetes $(\%)$ & $5 \%$ \\
\hline Hypertension $(\%)^{\mathrm{b}}$ & $42 \%$ \\
\hline \multicolumn{2}{|c|}{ Number of incident endpoints during follow-up } \\
\hline Acute coronary events ${ }^{\mathrm{c}}$ & 13,700 \\
\hline Stroke $^{\mathrm{d}}$ & 8,400 \\
\hline Heart failure ${ }^{\mathrm{e}}$ & 3,800 \\
\hline Atrial fibrillation ${ }^{\mathrm{f}}$ & 2,900 \\
\hline Type 2 diabetes $^{\mathrm{g}}$ & 3,400 \\
\hline Overall Death $^{\mathrm{h}}$ & 35,600 \\
\hline
\end{tabular}

Number are given for distinct individuals, excluding repeated measurements

${ }^{\text {a }}$ Status as of June 2014

b Blood pressure $>140 / 90$ or under treatment

${ }^{c}$ Definite or possible myocardial infarction or coronary death, or unstable angina pectoris. The number is available for 33 cohorts, estimated for 2 cohorts. Excluding individuals with history of cardiovascular disease

d The number is available for 34 cohorts, estimated for 1 cohort. Excluding individuals with history of cardiovascular disease

e The number is available for 11 cohorts, estimated for 1 cohort. Excluding individuals with history of heart failure

f The number is available for 10 cohorts. Excluding individuals with history of atrial fibrillation

$\mathrm{g}$ The number is available for 13 cohorts, estimated for 1 cohort. Excluding individuals with history of type 1 or type 2 diabetes

h The number is available for 37 cohorts, given for 1 cohort

Table 4 Overall characteristics of the BiomarCaRE disease cohorts

\section{Characteristics}

Number of cohorts 4

Number of individuals

8,746

Years of baseline examinations

1996-2012

Men (\%)

73

Mean age (SD) at baseline (years)

$61.5(12.4)$

Smoker $(\%)$

23

Type 2 diabetes $(\%)$

19

69

Hypertension (\%)

The numbers represent individuals at baseline, excluding repeated measurements. Disease cohort Rome ACB not included as data not been harmonized to BiomarCaRE database yet

specific and sensitive biomarkers has been discussed by several groups [10, 16, 33, 34]. In addition, assessment of the transcriptome can identify disease-related mRNA signatures which might be translated into clinically useful biomarkers [22, 31, 37]. Apart from proteomics and transcriptomics approaches, metabolite profiling in large, well-phenotyped cohorts is very rapidly emerging in the cardiovascular field [23, 27, 28, 35] and provides access to a biomolecular repertoire not covered by other omics applications.

The novel biomarkers are disclosed by the academic and SME partners. The SMEs introduce the technology and guide the development of the innovative assays needed for the measurement of novel biomarkers. In addition, the SMEs optimise the required technology so that medium- to high-throughput measurements become possible. Assay validation has been carried out before incorporation by the BiomarCaRE laboratory. The following technologies are established to apply omics-based discovery results in population based and disease cohorts: a) A microparticle-

Table 3 Disease cohorts of BiomarCaRE

\begin{tabular}{|c|c|c|c|c|c|c|c|c|c|c|}
\hline \multirow[t]{2}{*}{ Study } & \multirow[t]{2}{*}{ Country } & \multirow[t]{2}{*}{ Size } & \multirow{2}{*}{$\begin{array}{l}\text { Follow-up time } \\
\text { (years) }\end{array}$} & \multicolumn{4}{|l|}{ Endpoints } & \multicolumn{3}{|c|}{ Biomaterial } \\
\hline & & & & $\begin{array}{l}\text { Total } \\
\text { mortality }\end{array}$ & $\begin{array}{l}\text { Cardiac } \\
\text { death }\end{array}$ & $\begin{array}{l}\text { Cardiovascular } \\
\text { death }\end{array}$ & $\begin{array}{l}\text { Cardiovascular } \\
\text { events }\end{array}$ & Serum & $\begin{array}{l}\text { EDTA } \\
\text { Plasma }\end{array}$ & DNA \\
\hline AtheroGene & Germany & 3,476 & 7.5 & 386 & 244 & 260 & 460 & $\bullet$ & $\bullet$ & $\bullet$ \\
\hline KAROLA & Germany & 1,204 & 10 & 184 & 91 & 103 & 162 & $\bullet$ & $\bullet$ & $\bullet$ \\
\hline APACE & Switzerland & 2,248 & 2.2 & 174 & 71 & 73 & 534 & $\bullet$ & $\bullet$ & - \\
\hline stenoCardia & Germany & 1,818 & 0.5 & 34 & 0 & 7 & 38 & - & - & - \\
\hline $\begin{array}{l}\text { Rome } \\
\text { ACB* }\end{array}$ & Italy & $500^{\mathrm{a}}$ & - & - & - & - & & $\bullet$ & $\bullet$ & - \\
\hline
\end{tabular}

The disease cohorts of the project are listed including information on cohort size, years of follow-up and availability of biomaterial. Endpoint cardiovascular death includes fatal cardiovascular events such as cerebrovascular diseases; Endpoint cardiovascular events is a combined endpoint of cardiovascular death and non-fatal myocardial infarction* Median follow-up time. KAROLA, Langzeiterfolge der Kardiologischen Anschlussheilbehandlung; APACE, Advantageous Predictors of Acute Coronary Syndromes Evaluation Study; stenoCardia, Study for evaluation of newly onset chest pain and rapid diagnosis of myocardial necrosis

${ }^{\text {a }}$ Data not been harmonized to BiomarCaRE database yet 
Table 5 Clinical trials of BiomarCaRE

Clinical trials of the project are listed including information on trial setting, size, intervention, duration and primary endpoint of trial. LIPID, The Long-Term Intervention with Pravastatin in Ischaemic Disease Study; JUPITER, The Justification for the Use of Statins in Primary Prevention: An Intervention Trial Evaluating Rosuvastatin Trial; WHS, The Women's Health Study; IBIS-2, Integrated Biomarker and Imaging Study2; hsCRP, high-sensitivity measured C-reactive protein

\begin{tabular}{|c|c|c|c|c|c|}
\hline Study & Setting & Size & Intervention & $\begin{array}{l}\text { Median follow } \\
\text { up time (years) }\end{array}$ & Primary endpoint \\
\hline LIPID & $\begin{array}{l}\text { Secondary } \\
\text { prevention }\end{array}$ & 9,014 & $\begin{array}{l}\text { Pravastatin } \\
\quad(40 \mathrm{mg})\end{array}$ & 6.1 & $\begin{array}{l}\text { CHD Death } \\
\text { Non-fatal MI }\end{array}$ \\
\hline JUPITER & $\begin{array}{l}\text { Primary } \\
\text { prevention }\end{array}$ & 17,802 & $\begin{array}{l}\text { Rosuvastatin } \\
\quad(20 \mathrm{mg})\end{array}$ & 1.9 & $\begin{array}{l}\text { MI } \\
\text { Stroke } \\
\text { Cardiovascular death } \\
\text { Hospitalization for } \\
\quad \text { UAP }\end{array}$ \\
\hline WHS & $\begin{array}{l}\text { Primary } \\
\text { prevention } \\
\text { (women only) }\end{array}$ & 39,876 & $\begin{array}{l}\text { Aspirin }(100 \mathrm{mg}) \\
\text { every other day }\end{array}$ & 10.1 & $\begin{array}{l}\text { Non-fatal MI } \\
\text { Non-fatal stroke } \\
\text { Cardiovascular death }\end{array}$ \\
\hline IBIS-2 & $\begin{array}{l}\text { Secondary } \\
\text { prevention }\end{array}$ & 330 & $\begin{array}{r}\text { Darapladib } \\
(160 \mathrm{mg})\end{array}$ & 1 & $\begin{array}{l}\text { Coronary atheroma } \\
\text { Progression by IVUS } \\
\text { Plaque deformability } \\
\text { by Palpography } \\
\text { hsCRP }\end{array}$ \\
\hline
\end{tabular}

Table 6 Number of cases and non-cases in the individual case cohort sets of the cohorts selected for the BiomarCaRE case-cohort study

\begin{tabular}{|c|c|c|c|c|c|c|c|}
\hline \multirow[t]{2}{*}{ Study } & \multirow[t]{2}{*}{ Country } & \multicolumn{5}{|c|}{ Number of incident cases } & \multirow{2}{*}{$\begin{array}{l}\text { Number of } \\
\text { non-cases }\end{array}$} \\
\hline & & $\begin{array}{l}\text { Coronary heart } \\
\text { disease }\end{array}$ & Stroke & $\begin{array}{l}\text { Heart } \\
\text { failure }\end{array}$ & $\begin{array}{l}\text { Atrial } \\
\text { fibrillation }\end{array}$ & Type 2 diabetes & \\
\hline Glostrup & Denmark & 821 & 744 & 608 & 603 & 526 & 2,209 \\
\hline ATBC Placebo & Finland & 1,414 & 822 & - & - & - & 2,068 \\
\hline FINRISK 97 & Finland & 354 & 260 & 442 & 307 & 525 & 1,384 \\
\hline PRIME France & France & 286 & 90 & - & - & - & 414 \\
\hline KORA S3/S4 & Germany & 252 & 224 & - & - & 390 & 910 \\
\hline Brianza & Italy & 218 & 119 & - & - & - & 369 \\
\hline PRIME Belfast & United Kingdom & 185 & 53 & - & - & - & 282 \\
\hline SHHEC & Scotland & 940 & 411 & 489 & 475 & 477 & 2,055 \\
\hline Total & 4,470 & 2,723 & 1,539 & 1,385 & 1,918 & 9,691 & \\
\hline
\end{tabular}

Numbers are given as status in June 2014. ATBC, Alpha-Tocopherol, Beta-Carotene Cancer Prevention Study; FINRISK, FINRISK Study, Glostrup, Glostrup Study; SHHEC, Scottish Heart and Health Extended Cohorts; PRIME, Prospective Epidemiological Study of Myocardial Infarction Study; KORA, Kooperative Gesundheitsforschung in der Region Augsburg; Brianza, The Brianza Study

based protein biomarker panel will be validated in the disease cohorts to confirm their value as markers in the prediction of secondary manifestations of CVD [16]. For this purpose, development of a 4-plex assay based on a Luminex platform is ongoing An Enzyme-Linked Immunosorbent Assay (ELISA) is being developed within BiomarCaRE to assess cardiovascular risk, specifically in women. To investigate circulating miRNAs, a highly versatile multiplex detection platform to support the multiplex measurement of circulating miRNAs is being developed. Candidate genes from transcriptomics datasets of
BiomarCaRE partners [38] that may be related to cardiovascular events or phenotypes have been selected as biomarker targets, and ELISAs for higher throughput measurements are being developed. The AbsoluteIDQ p180 kit (www.biocartes.com), which identifies and quantifies 185 metabolites by mass spectrometry, will be used to profile the metabolome. The metabolome profile and the transcriptomics-based biomarkers including miRNA will be assessed within BiomarCaRE in the case cohort setting $(n=20,000)$. Figure 3 provides an overview of established and emerging biomarkers measured in BiomarCaRE. 


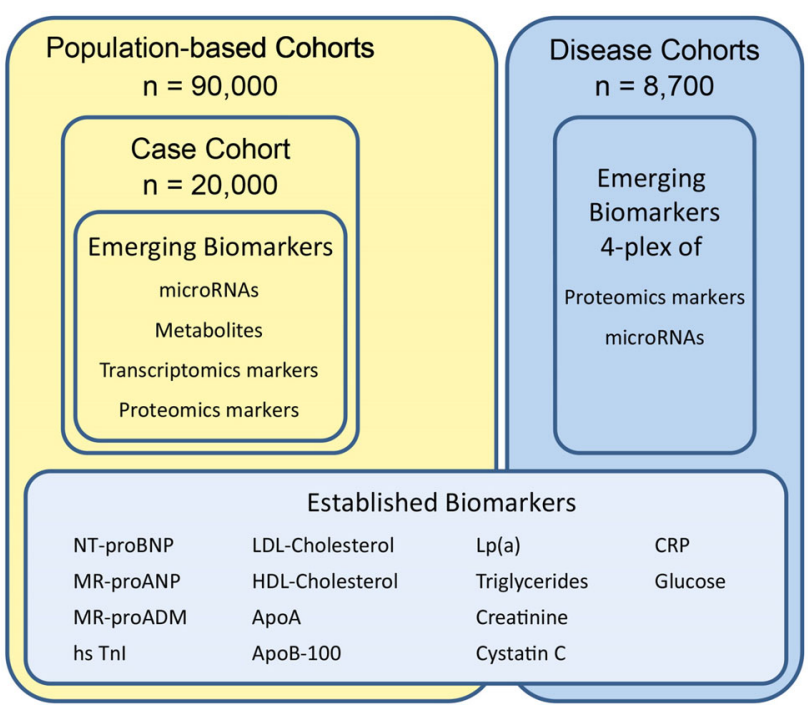

Fig. 3 Emerging and established biomarkers measured in phase 1 of the population-based cohorts (including a case-cohort set) and the disease cohorts within BiomarCaRE. NTproBNP, N-terminal pro B-type natriuretic peptide, MR-proANP mid-regional pro atrial natriuretic papetide; MR-proADM, mid-regional pro adrenomedullin; hsTnI, high-sensitivity assayed Troponin I; ApoA, apolipoprotein A1; ApoB100, apolipoprotein B100; Lp(a) Lipoprotein a; CRP, C-reactive protein

In addition to the emerging, omics-based biomarkers, novel biomarkers from the literature, such as biomarkers for heart failure (e.g. soluble isoforms of the IL-1 receptor family member (sST2) and Galectin-3) are being selected. An overview of all established and emerging, literaturebased biomarkers to be measured in BiomarCaRE is given in the Supplementary Material.

\section{Measurement of biomarkers}

In Module 2 of the project (Fig. 2), a two-phase approach for the biomarker measurement is used: in phase 1, established and novel biomarkers are determined in a set of population cohorts from different European regions, including 90,000 subjects with 6,000 incident acute coronary events, 3,800 incident strokes, 3,500 incident cases of heart failure, 2,600 cases with incident atrial fibrillation and 2,800 incident type 2 diabetes diagnoses during followup (Table 1). In addition, markers which are considered as most innovative but "high risk" markers, such as metabolites, miRNA and other novel markers derived from omics approaches will be measured in a case-cohort set of up to 20,000 population-based subjects including 4,500 incident acute coronary events. This case-cohort design reduces the number of biomarker measurements without substantially reducing statistical power [19]. The case-cohort study involves a random subsample of the selected population cohorts and in addition all incident cases of these cohorts
(Table 6). Independent of the population-based cohorts, the selected biomarkers are also measured in the four diseased cohorts $(\mathrm{n}=8,746$, Table 3 ).

In phase 2, the most promising biomarkers from phase 1 are further determined in 130,000 subjects from European population cohorts (Table 1). Biomarkers measured within the BiomarCaRE project are depicted in Fig. 3.

\section{Statistical concepts}

BiomarCaRE comprises two closely interacting data centres: one centre that is responsible for the analyses of all population-derived biomarkers which is based at the National Institute for Health and Welfare (THL) in Helsinki; Finland. This data centre has a long-standing and unparalleled experience of epidemiological data harmonization and central data management and analyses of the MORGAM Project [9] and its predecessor, the WHO MONICA Project (Tunstall-Pedoe and Project [32]. The second data centre is based at the University at Lübeck, Germany and is responsible for the analyses of biomarkers derived from the diseased cohorts. Data obtained in clinical trial repositories will be analysed directly at the respective partner site. Figure 4 illustrates and summarizes analyses performed in phases 1 and 2 .

As biomarkers may have different effects in healthy and diseased subjects, the population-based and diseased cohorts will be analysed separately. The statistical analysis for both types of cohorts will begin with univariate testing of each biomarker with adjustment for classical cardiovascular risk factors. Significant biomarkers are selected for subsequent analysis in phase 2 cohorts for validation.

The risk models are developed using Cox regression analysis, taking the case-cohort sampling into account [19] for some biomarkers. The modelling steps include initial checking of model assumptions and the stability and parsimony of biomarker selection. As a complementary approach for biomarker selection, we will apply random survival forests [13, 15] which is a machine learning technique to determine important variables for the prediction of individual survival times. Performance of the risk estimation models will be cross-validated using calibration, discrimination indices and graphs and net reclassification improvement. The developed predictive models are compared with established risk score models, such as the SCORE [6].

The impact of the biomarkers and the predictive models developed in phase 1 is externally validated using the prospective population-based cohorts and biomarker data from phase 2 . These analyses will result in the construction of a risk model including classical risk factors and a combination of the validated biomarkers (BiomarCaRE Panel). As a secondary subsidiary aspect, the cross- 
Fig. 4 Overview of data analyses performed in phases 1 and 2 of BiomarCaRE

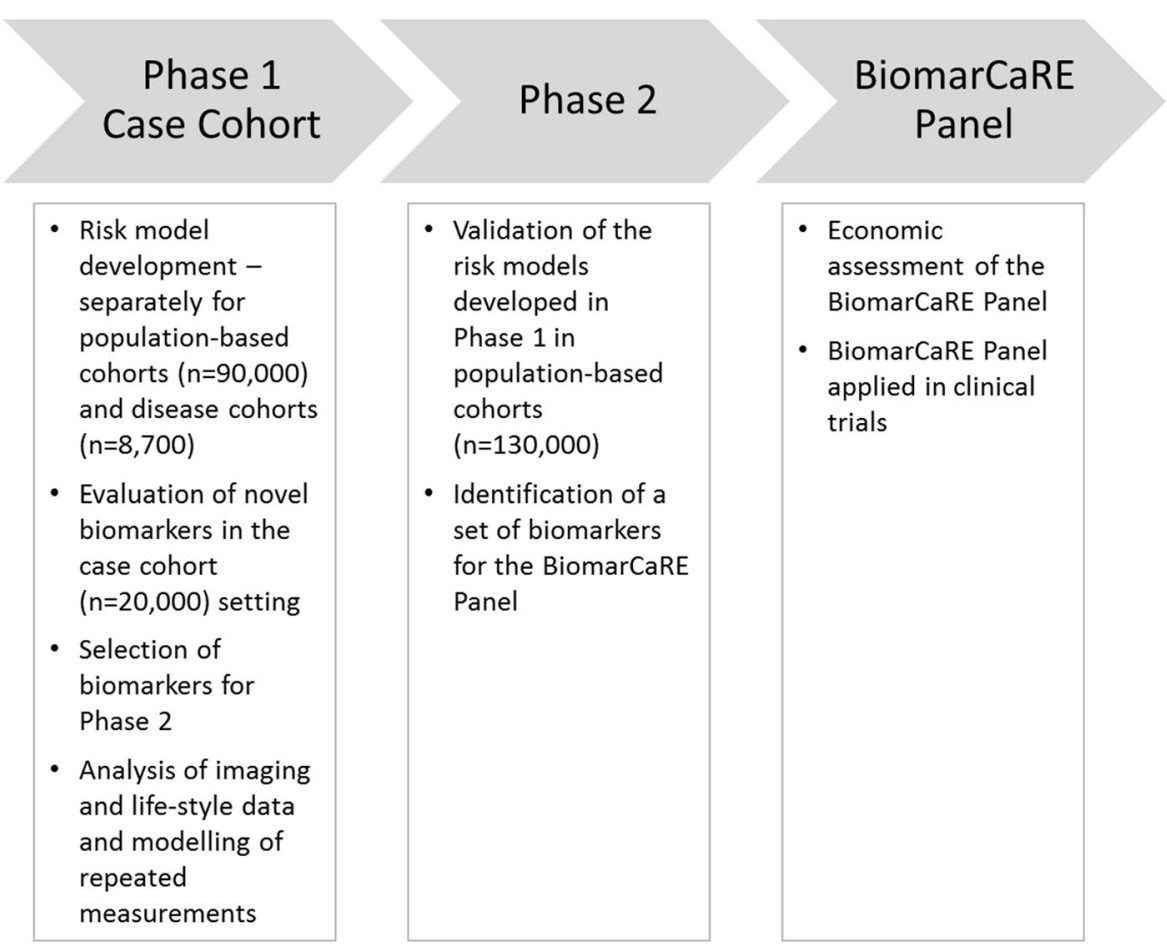

sectional association between biomarkers and cardiac imaging data and between biomarkers and health behaviour data will be assessed in individual studies where data are available. Furthermore, joint modelling of repeated measurements of the biomarkers and survival data will be performed for selected cohorts.

To analyze the effects of various pharmacological interventions on the BiomarCaRE panel of selected biomarkers stepwise statistical strategies will be applied in the clinical trials.

In a first step, the predictive value of the BiomarCaRE panel will be assessed in samples from the clinical trials, adjusted for medication and stratified by intervention group. In a second step, the change in biomarker levels from baseline to (on average) 1 year on treatment will be assessed. Finally, the treatment effect will be analyzed according to biomarker levels at baseline (high vs. low).

\section{Proposed imputation strategy for the BiomarCaRE project}

Clinical and biomarker variables are often incomplete due to e.g. sample unavailability or assay failure. Data analysis using only complete sets for multiple variables would lead to loss of available information, thus to reduced statistical power. Furthermore, the results of such analysis can be biased. One approach to make the best use of available data is to predict the missing information from the observed data. This process minimizes potential bias, and is termed multiple imputation [8] and will be used in BiomarCaRE analysis when appropriate. A common strategy in clinical epidemiology is to exclude variables with more than $20 \%$ missing data because of the high potential for a severe bias. If less than $5 \%$ of the data are missing, substantial bias is unlikely [2]. If $20 \%$ of the biomarker measurements are missing, the biomarker may be excluded from a multimarker analysis, depending on what is known about the reason for the missing data. Decisions will be made on a case by case basis.

Economic assessment of single and combined biomarker application

In the third module of the project (Fig. 2), the focus will be on the clinical utilisation and cost-effectiveness of the selected biomarkers, and their single and joint application. Selected biomarkers from phase 2 will be measured in samples of clinical trials to test their interaction with risk lowering therapy (Table 5) and a decision-analytic model will be developed to estimate long-term effectiveness and cost-effectiveness of a primary or secondary preventive strategy based on additional risk assessment using these biomarkers.

Decision-analytic modelling allows for the combination of empirical evidence on improvements in risk prediction and published evidence on short- and long-term effects of health are interventions to estimate the health effects on patient-relevant endpoints such as life expectancy or quality-adjusted life expectancy. Total costs of a strategy will then be estimated by combining cost savings through prevention of advanced disease and costs for the biomarker 
panel tests, diagnostic tests and work-up, drug treatment and monitoring of patients. A decision-analytic state-transition (Markov) model will be used, as this model type suits decision problems that can be described in specific health states and transition probabilities [5]. The analysis will be performed as a cohort simulation or as a microsimulation depending on the number of covariates to be tracked in the simulation [30], and deterministic and probabilistic sensitivity analyses will be performed. The long-term effectiveness of the biomarker-guided strategies will be expressed in life years and quality-adjusted life years (QALY). Cost-effectiveness will be expressed as incremental cost-effectiveness ratios, that is, the additional costs divided by gains in effectiveness [29]. In particular, we will systematically vary biomarkers and cut-offs in our model to identify candidate strategies for effective and costeffective biomarker testing that can be investigated in future clinical trials.

\section{Discussion}

The BiomarCaRE project aims to determine the additional value of multiple (new) biomarkers to improve risk estimation of CVD related events in Europe. The project is unique in terms of its dimension, targeting of novel biomarkers based on -omics technology, and the evaluation of the impact of a multiple biomarker score in large prospective population cohorts across different European regions. The project integrates modern, mainly SME driven molecular technologies with epidemiological approaches supplemented by economical assessment. Evaluation of biomarkers on a large-scale using high sensitivity tests improves the precision of the risk estimates. A major strength of the project is the derivation of novel risk estimation models and assessment in a variety of settings ranging from general populations to those with disease and hypothesis-generating clinical trials. The resulting models may prove most useful in refining risk in subgroups of the population that could benefit from early treatment.

To date, it is not clear to what extent single or multiple biomarkers improve CVD prediction and how they provide additional information for therapy selection and guidance. Ideally, selection of biomarkers and generation of multimarker panels would be based both on biological plausibility (and independent causal effects) and empirical evidence on their influence on disease risk. However, the causal relevance of many predictive biomarkers of CVD has yet to be fully established, but this may not necessarily be required for risk prediction. Recent research has demonstrated that scores comprising biomarkers that show low to moderate correlation between each other and represent different biological pathways could improve discrimination and calibration $[4,14,25]$. As the number of pathways known to contribute to cardiovascular risk is expanding, selection of biomarkers from different pathways is challenging. The added value contributed by the BiomarCaRE consortium is the ability to capitalise on proteomics, metabolomics, transcriptomics and miRNAomics approaches which may yield promising candidate markers from novel pathways which can be compared robustly to other established and emerging biomarkers. This is based on newly developed technologies to apply innovative biomarkers on a large-scale basis. Furthermore, the project will extend our knowledge of biomarker-driven prognostication to encompass the wider cost-effectiveness implications using a simulation approach.

The ultimate goal of BiomarCaRE, building a robust European risk score, would greatly facilitate individualized risk estimation, screening, and diagnosis of CVD. The novel risk score would carry the potential to change existing guidelines and could thus have significant public health impact.

Current research further suggests that considerable differences exist between women and men with respect to disease mechanisms and outcomes. However, small number of women developing manifest disease in population studies has often made it difficult to assess sex differences. Furthermore, women are not adequately represented in clinical trials. Thus, gender-specific analyses have been mostly underpowered. Because of the increasing incidence of CVD in women, there is a need to gain insight into gender-specific biomarker properties. Within BiomarCaRE the large cohort size will allow gender-based subgroup analyses with sufficient power.

\section{Expected outcome and perspective of personalized medicine in Europe}

The BiomarCaRE project will be in a unique position to carefully design pragmatic randomized controlled trials to understand the clinical implications of reclassification, in particular to establish the safety and effectiveness of deferring treatment for those reclassified into a lower risk category based on biomarkers. Further, the study will give clues to whether any intensified intervention in those reclassified to higher risk categories might ultimately result in improved clinical outcomes. In this regard, BiomarCaRE has already initiated the planning of the "post" BiomarCaRE phase that will prospectively validate clinical utilisation in personalised medicine, by proposing the execution of a biomarker guided clinical trial utilizing the results of the BiomarCaRE consortium.

Acknowledgments This work has been supported by the European Union Seventh Framework Programme (FP7/2007-2013) under grant 
agreement No. HEALTH-F2-2011-278913 (BiomarCaRE). Funding and acknowledgments of each study is provided in the Supplementary Material.

Conflict of interest Abbott Diagnostics provides test reagents for ApoA1, ApoB100, Triglycerides, HDL-Cholesterol, LDL-Cholesterol, high-sensitivity Troponin I, C-reactive Protein, Lp(a), Creatinine, and Glucose.. The authors declare that they have no conflict of interest.

Open Access This article is distributed under the terms of the Creative Commons Attribution License which permits any use, distribution, and reproduction in any medium, provided the original author(s) and the source are credited.

\section{Members of the BiomarCaRE study group}

Medical University Hamburg Eppendorf, Hamburg, Germany: Stefan Blankenberg, Tanja Zeller, Simone Schnella, Renate B Schnabel, Francisco Ojeda, Sebastian Appelbaum, Johannes Neumann; Mahir Karakas, Christian Schulte, Annika Jagodzinski

National Institute for Health and Welfare, Helsinki, Finland: Kari Kuulasmaa, Veikko Salomaa, Jarmo Virtamo, Satu Männistö, Matti Niemelä, Tarja Tuovinen Zygimantas Cepaitis, Ari Haukijärvi, Aki Havulinna, Olli Saarela, Jukka Kontto, Arto Pietilä

Cavadis B.V., Utrecht, The Netherlands: Heico Breek, Herfita Agustiandari

Universitair Medisch Centrum, Utrecht, The Netherlands: Gerard Pasterkamp, Hester den Ruijter, Marten Siemelink

Helmholtz Zentrum Muenchen, Germany: Barbara Thorand, Annette Peters

Biocrates Life Sciences AG, Innsbruck, Austria: Cornelia Röhring; Manuel Kratzke

University Lübeck, DE: Andreas Ziegler, Arne Schillert, Andrea Senft

University for Health Sciences, Medical Informatics and Technology, Hall i.T., Austria: Annette Conrads-Frank, Uwe Siebert, Petra Schnell-Inderst, Felicitas Kühne

Medizinische Hochschule Hannover, Germany: Kai C. Wollert, Tibor Kempf

Fleet Bioprocessing Ltd., Hartley Wintney, UK: Alastair Dent, Julian Duncan, Nic Christofides, Lindsay Robertson

Biocartis SA, Lausanne, Switzerland: Eva Servoli, Patrick van den Bogaard

Research Network Services Ltd., Berlin, Germany: Eric Werner

Universitätsmedizin der Johannes Gutenberg-Universität Mainz, Germany: Philipp Wild, Karl Lackner, Thomas Münzel

University of Troms $\varnothing$, Norway: Ellisiv B Mathiesen, Inger Njølstad
Università Cattolica del Sacro Cuore, Roma, Italy: Luigi Marzio Biasucci

IRCCS Istituto Neurologico Mediterraneo Neuromed, Pozzilli, Italy: Licia Iacoviello, Simona Costanzo, Augusto Di Castelnuovo, Giovanni de Gaetano

Ernst-Moritz-Arndt Universität Greifswald, Germany: Henry Völzke, Roberto Lorbeer

University College London, UK: Martin Bobak

Institute Pasteur de Lille, France: Jean Dallongeville, Jean Ferrières, Dominique Arveiler, Philippe Amouyel

Catalan Institute of Cardiovascular Sciences, Barcelona, Spain: Susana Sans

The Queen's University of Belfast, UK: Frank Kee, Maria Hughes, Felicity Lamrock, Karen Cairns

Umeå Universitet, Sweden:Stefan Söderberg

Research Centre for Prevention and Health, Glostrup, Denmark: Torben Jørgensen, Allan Linneberg, Anders Borglykke

University of Insubria, Varese, Italy: Marco Ferrario, Giovanni Veronesi

University of Dundee, UK: Hugh Tunstall-Pedoe, Jill Belch, Gwen Kennedy

Universitätsspital Basel, Switzerland: Christian Müller, Raphael Twerenbold, Beate Hartmann

Universitätsklinikum Ulm, Germany: Wolfgang Koenig, Dietrich Rothenbacher

Hamilton Health Sciences Corporation, Canada: Sonia Anand, Salim Yusuf

University of Sydney, Sydney, Australia: John Simes, Andrew Tonkin

Harvard Medical School, Brigham and Women's Hospital, Boston, USA: Brendan Everett, Paul Ridker

\section{References}

1. Anand IS, Kempf T, Rector TS, Tapken H, Allhoff T, Jantzen F, Kuskowski M, Cohn JN, Drexler H, Wollert KC. Serial measurement of growth-differentiation factor-15 in heart failure: relation to disease severity and prognosis in the Valsartan Heart Failure Trial. Circulation. 2010;122(14):1387-95.

2. Arbuckle JL. Full information estimation in the presence of incomplete data. In: Marcoulides GA, Schumaker RE, editors. Advanced structural equation modeling: Issues and techniques. Mahwah, NJ: Lawrence Erlbaum Associates; 1996. p. 243-277.

3. Assmann G, Cullen P, Schulte H. Simple scoring scheme for calculating the risk of acute coronary events based on the 10-year follow-up of the prospective cardiovascular Munster (PROCAM) study. Circulation. 2002;105(3):310-5.

4. Blankenberg S, Zeller T, Saarela O, Havulinna AS, Kee F, Tunstall-Pedoe H, Kuulasmaa K, Yarnell J, Schnabel RB, Wild PS, Munzel TF, Lackner KJ, Tiret L, Evans A, Salomaa V, M. Project. Contribution of 30 biomarkers to 10 -year cardiovascular risk estimation in 2 population cohorts: the MONICA, risk, genetics, archiving, and monograph (MORGAM) biomarker project. Circulation. 2010;121(22):2388-97. 
5. Caro JJ, Briggs AH, Siebert U, Kuntz KM, Force I-SMGRPT. Modeling good research practices-overview: a report of the ISPOR-SMDM Modeling Good Research Practices Task Force-1. Value Health. 2012;15(6):796-803.

6. Conroy RM, Pyorala K, Fitzgerald AP, Sans S, Menotti A, De Backer G, De Bacquer D, Ducimetiere P, Jousilahti P, Keil U, Njolstad I, Oganov RG, Thomsen T, Tunstall-Pedoe H, Tverdal A, Wedel H, Whincup P, Wilhelmsen L, Graham IM, S. p. group. Estimation of ten-year risk of fatal cardiovascular disease in Europe: the SCORE project. Eur Heart J. 2003;24(11):987-1003.

7. D'Agostino RB Sr, Vasan RS, Pencina MJ, Wolf PA, Cobain M, Massaro JM, Kannel WB. General cardiovascular risk profile for use in primary care: the Framingham Heart Study. Circulation. 2008;117(6):743-53.

8. Rubin DB. Multiple imputation for nonresponse in surveys. New York, NY: Wiley; 1987.

9. Evans A, Salomaa V, Kulathinal S, Asplund K, Cambien F, Ferrario M, Perola M, Peltonen L, Shields D, Tunstall-Pedoe H, Kuulasmaa K, M. Project. MORGAM (an international pooling of cardiovascular cohorts). Int J Epidemiol. 2005;34(1):21-7.

10. Fichtlscherer S, Zeiher AM, Dimmeler S. Circulating microRNAs: biomarkers or mediators of cardiovascular diseases? Arterioscler Thromb Vasc Biol. 2011;31(11):2383-90.

11. Fox KA, Carruthers KF, Dunbar DR, Graham C, Manning JR, De Raedt H, Buysschaert I, Lambrechts D, Van de Werf F. Underestimated and under-recognized: the late consequences of acute coronary syndrome (GRACE UK-Belgian Study). Eur Heart J. 2010;31(22):2755-64.

12. Gerszten RE, Asnani A, Carr SA. Status and prospects for discovery and verification of new biomarkers of cardiovascular disease by proteomics. Circ Res. 2011;109(4):463-74.

13. Ishwaran H, Kogalur UB, Blackstone EH, Lauer MS. Random survival forests. Ann Appl Statist. 2008;2:841-860.

14. Herder C, Karakas M, Koenig W. Biomarkers for the prediction of type 2 diabetes and cardiovascular disease. Clin Pharmacol Ther. 2011;90(1):52-66.

15. Hothorn T, Buhlmann P, Dudoit S, Molinaro A, van der Laan MJ. Survival ensembles. Biostatistics. 2006;7(3):355-73.

16. Kanhai DA, Visseren FL, van der Graaf Y, Schoneveld AH, Catanzariti LM, Timmers L, Kappelle LJ, Uiterwaal CS, Lim SK, Sze SK, Pasterkamp G, de Kleijn DP, S. S. Group. Microvesicle protein levels are associated with increased risk for future vascular events and mortality in patients with clinically manifest vascular disease. Int J Cardiol. 2013;168(3):2358-63.

17. Keller T, Zeller T, Peetz D, Tzikas S, Roth A, Czyz E, Bickel C, Baldus S, Warnholtz A, Frohlich M, Sinning CR, Eleftheriadis MS, Wild PS, Schnabel RB, Lubos E, Jachmann N, Genth-Zotz S, Post F, Nicaud V, Tiret L, Lackner KJ, Munzel TF, Blankenberg S. Sensitive troponin I assay in early diagnosis of acute myocardial infarction. N Engl J Med. 2009;361(9):868-77.

18. Khot UN, Khot MB, Bajzer CT, Sapp SK, Ohman EM, Brener SJ, Ellis SG, Lincoff AM, Topol EJ. Prevalence of conventional risk factors in patients with coronary heart disease. JAMA. 2003; 290(7):898-904.

19. Kulathinal S, Karvanen J, Saarela O, Kuulasmaa K. Case-cohort design in practice-experiences from the MORGAM Project. Epidemiol Perspect Innov. 2007;4:15.

20. Kulathinal S, Niemelä M, Kuulasmaa K. Contributors from Participating Centres, for the MORGAM Project. Description of MORGAM Cohorts. MORGAM Project e-publications. Internet 2005.

21. Melander O, Newton-Cheh C, Almgren P, Hedblad B, Berglund G, Engstrom G, Persson M, Smith JG, Magnusson M, Christensson A, Struck J, Morgenthaler NG, Bergmann A, Pencina MJ, Wang TJ. Novel and conventional biomarkers for prediction of incident cardiovascular events in the community. JAMA. 2009;302(1):49-57.
22. Pedrotty DM, Morley MP, Cappola TP. Transcriptomic biomarkers of cardiovascular disease. Prog Cardiovasc Dis. 2012;55(1):64-9.

23. Rhee EP, Gerszten RE. Metabolomics and cardiovascular biomarker discovery. Clin Chem. 2012;58(1):139-47.

24. Ridker PM, Danielson E, Fonseca FA, Genest J, Gotto A M Jr, Kastelein JJ, Koenig W, Libby P, Lorenzatti AJ, MacFadyen JG, Nordestgaard BG, Shepherd J, Willerson JT, Glynn RJ, J. S. Group. Rosuvastatin to prevent vascular events in men and women with elevated C-reactive protein. $\mathrm{N}$ Engl $\mathrm{J}$ Med. 2008;359(21):2195-207.

25. Salomaa V, Havulinna A, Saarela O, Zeller T, Jousilahti P, Jula A, Muenzel T, Aromaa A, Evans A, Kuulasmaa K, Blankenberg $\mathrm{S}$. Thirty-one novel biomarkers as predictors for clinically incident diabetes. PLoS ONE. 2010;5(4):e10100.

26. Schnabel RB, Schulz A, Messow CM, Lubos E, Wild PS, Zeller T, Sinning CR, Rupprecht HJ, Bickel C, Peetz D, Cambien F, Kempf T, Wollert KC, Benjamin EJ, Lackner KJ, Munzel TF, Tiret L, Vasan RS, Blankenberg S. Multiple marker approach to risk stratification in patients with stable coronary artery disease. Eur Heart J. 2010;31(24):3024-31.

27. Shah SH, Bain JR, Muehlbauer MJ, Stevens RD, Crosslin DR, Haynes C, Dungan J, Newby LK, Hauser ER, Ginsburg GS, Newgard CB, Kraus WE. Association of a peripheral blood metabolic profile with coronary artery disease and risk of subsequent cardiovascular events. Circ Cardiovasc Genet. 2010;3(2):207-14.

28. Shah SH, Kraus WE, Newgard CB. Metabolomic profiling for the identification of novel biomarkers and mechanisms related to common cardiovascular diseases: form and function. Circulation. 2012;126(9):1110-20.

29. Siebert U. Cost-effectiveness. New York: McGraw-Hill; 2007.

30. Siebert U, Alagoz O, Bayoumi AM, Jahn B, Owens DK, Cohen DJ, Kuntz KM. State-transition modeling: a report of the ISPORSMDM Modeling Good Research Practices Task Force-3. Med Decis Making. 2012;32(5):690-700.

31. Thomas GS, Voros S, McPherson JA, Lansky AJ, Winn ME, Bateman TM, Elashoff MR, Lieu HD, Johnson AM, Daniels SE, Ladapo JA, Phelps CE, Douglas PS, Rosenberg S. A blood-based gene expression test for obstructive coronary artery disease tested in symptomatic nondiabetic patients referred for myocardial perfusion imaging the COMPASS study. Circ Cardiovasc Genet. 2013;6(2):154-62.

32. Tunstall-Pedoe H, Kuulasmaa K, Tolonen H, Davidson M, Mendis S with and o. c. f. T. W. M. Project. MONICA, Risk, Genetics, Archiving, and Monograph. MONICA Monograph and Multimedia Sourcebook. T.-P. H, Geneva: World Health Organization; 2003.

33. van Rooij E. The art of microRNA research. Circ Res. 2011;108(2):219-34.

34. Wang JW, Gijsberts CM, Seneviratna A, de Hoog VC, Vrijenhoek JE, Schoneveld AH, Chan MY, Lam CS, Richards AM, Lee CN, Mosterd A, Sze SK, Timmers L, Lim SK, Pasterkamp G, de Kleijn DP. Plasma extracellular vesicle protein content for diagnosis and prognosis of global cardiovascular disease. Neth Heart J. 2013;21(10):467-71.

35. Wang TJ, Larson MG, Vasan RS, Cheng S, Rhee EP, McCabe E, Lewis GD, Fox CS, Jacques PF, Fernandez C, O'Donnell CJ, Carr SA, Mootha VK, Florez JC, Souza A, Melander O, Clish $\mathrm{CB}$, Gerszten RE. Metabolite profiles and the risk of developing diabetes. Nat Med. 2011;17(4):448-53.

36. Yusuf S, Hawken S, Ounpuu S, Dans T, Avezum A, Lanas F, McQueen M, Budaj A, Pais P, Varigos J, Lisheng L, Investigators IS. Effect of potentially modifiable risk factors associated with myocardial infarction in 52 countries (the INTERHEART study): case-control study. Lancet. 2004;364(9438):937-52.

37. Zeller T, Blankenberg S. Blood-based gene expression tests: promises and limitations. Circ Cardiovasc Genet. 2013;6(2):139-40. 
38. Zeller T, Wild P, Szymczak S, Rotival M, Schillert A, Castagne R, Maouche S, Germain M, Lackner K, Rossmann H, Eleftheriadis M, Sinning CR, Schnabel RB, Lubos E, Mennerich D, Rust W, Perret C, Proust C, Nicaud V, Loscalzo J, Hubner N, Tregouet D, Munzel T, Ziegler A, Tiret L, Blankenberg S, Cambien F. Genetics and beyond-the transcriptome of human monocytes and disease susceptibility. PLoS ONE. 2010;5(5):e10693.
39. Zethelius B, Berglund L, Sundstrom J, Ingelsson E, Basu S, Larsson A, Venge P, Arnlov J. Use of multiple biomarkers to improve the prediction of death from cardiovascular causes. N Engl J Med. 2008;358(20):2107-16. 